\title{
DESARROLLO URBANO DE ALICANTE: LA VILA NOVA MEDIEVAL
}

\author{
A. Ramos Hidalgo \\ Departamento de Geografía Humana \\ Universidad de Alicante
}

\section{INTRODUCCION}

El pasado medieval de la ciudad de Alicante no resulta conocido de manera satisfactoria en la actualidad, pese al empeño de los autores que, con mayor o menor fortuna, se han ocupado, en distintas épocas y con diversos métodos, de la temática alicantina, dada la notoria escasez de fuentes utilizables a tal fin, no obstante haberse producido en el referido período un importante proceso fundacional urbano, semejante a los realizados en el Levante español durante el proceso de formación de los reinos de la Corona de Aragón.

Sin embargo, en los últimos años nuevas publicaciones han enriquecido el limitado conocimiento que existía acerca de la historia de la ciudad, de su población y de las formas económicas adoptadas con anterioridad en la misma (1), entre las que destacamos en particular, en lo referente a la morfología urbana, la excelente aportación del arquitecto M. Beviá (2), que resuelve de manera convincente los problemas

(1) Destaca, a este fin, la vasta serie de publicaciones de V. MARTínEZ MORELLA, J. M. DEL ESTAL y las de J. TORRES FONTES por la cuantiosa documentación aportada y $P$. $M$. ORTS Y BOSCH, Alicante, notes historicas. 1373-1800. Valencia, 1971.

(2) M. BEVIA, «formació del País Valencia i canvi urba: el cas d'Alacant». L'ESPILL, 15, Valencia, 1982, pp. 53-82. 
de la localización y extensión del núcleo musulmán, al tiempo que cartografía el área de la vila nova, desarrollada a partir de la Conquista y asentamiento de repobladores cristianos, y define, por último, los procesos de ocupación de los espacios intersticiales existentes entre ésta y la primitiva vila vella musulmana.

Puede afirmarse que en las postrimerías de su período islámico la pequeña ciudad asentada en la vertiente meridional del cerro Benacantil, se vio implicada en el contexto general de anarquía dominante en el SE. peninsular. Sometida al dominio de Zayyan, desde abril de 1239, Alicante registra las alternativas políticas propias de este momento: el rechazo generalizado a dicho caudillo, el cual, tras ser depuesto en el verano de 1241 por Ibn Hud, se refugió en la ciudad (Lakant - al Hosun) de donde sería expulsado por Alfonso el Sabio, en el período que media entre el 18 de mayo de 1247 y el 7 del mismo mes 1248, al no aceptar éste las estipulaciones de Alcaraz firmadas entre el Rey Sabio -en nombre de su padre- e Ibn Hud (3), a causa de lo cual, al ser rechazadas las condiciones de protección castellana, según refiere Ibn Jaldum (4), la ciudad fue tomada militarmente por Alfonso el Sabio que le confirió la institución concejil, acción jurídica real que de ordinario se daba a las ciudades musulmanas tomadas por las armas (5).

\section{EL REPARTIMIENTO Y LA REPOBLACION}

Tras la Conquista de Alfonso el Sabio realizó un primer asentamiento de cristianos (6) y, a continuación, una primera partición concluida antes de 1252, cuando amplió su término municipal con las siete aldeas de Novelda, Aspe el Viejo, Aspe el Nuevo, Nompot (Monforte), Agost, Busot y Aguas (7), y a éste siguió otro segundo repartimiento antes de 1258 (8), realizado por los repartidores reales D. Durant de Plazencia,

(3) J. M. DEL ESTAL, «Conquista y repoblación de Orihuela y Alicante por Alfonso el Sabiow, R.I.E.A., 33, p. 77.

(4) IBN KHALDOUN, Histoire des berbères et des dynasties musulmanes de l'Afrique septentrionales, p. 312.

(5) Cf. V. GAUTIER DALCHE, Historia urbana de León y Castilla en la Edad Media, siglos IX-XIII, p. 162 y ss.

(6) J. M. DEL ESTAL; op. cit., p. 82.

(7) V. MARTINEZ MORELLA, Privilegios y franquezas de Alfonso $X$ el Sabio a Alicante, $p$. 9; AMA, Arm. I, L. 3, fol. 298v., (Doc. 29-VIII-1253).

(8) J. TORRES FONTES, CODOM III dto. XLVI p. 64, Valladolid 10, IV, 1258, vid. nota 5. 
Garci Ferrández y Bernat Ferrer, confeccionando el libro del Repartimiento de Alicante, cuyo paradero se desconoce, probablemente desaparecido en los conflictos de la guerra castellano aragonesa entre los dos Pedros, como testimonia Pedro IV de Aragón (9).

Nada sabemos de las consecuencias que acarrearía a la población musulmana el proceso de asentamiento cristiano en relación con su permanencia o expulsión de la ciudad, aunque es de suponer que el número de musulmanes afectados inicialmente de destierro no sería más que una minoria, porque el monarca castellano consciente del insustituible papel de la mano de obra mudéjar, pidió al concejo y a su almojarife, D. Berenguel de Moncada, que nadie avasallase o actuase injustamente contra los musulmanes (10).

El asentamiento cristiano debió ser al principio de orden militar (11), para la defensa armada del castillo y la ciudad agregándose después -en cantidades no cuantificables- gentes de las distintas capas sociales y profesionales a las que se entregó casas y bienes inmuebles, urgiéndoles a que habitaran la ciudad y favoreciéndoles con exenciones (12) tales como portazgo de pan y del vino, del depósito obligatorio en el Alfolí, de servicios armados y pagos comerciales, beneficiando a los comerciantes locales sobre los forasteros o extranjeros, a la vez que ofrecía recursos financieros al Concejo para «... cercar la villa, alabrarla, et pora carreras...." (13). De idéntico modo, para el reforzamiento de sus murallas aportó 2.000 maravedís chicos, y para la traída y conducción de agua potable desde los manantiales de la Fuensanta al interior de la ciudad entregó 500 (14), autorizando la construcción de molinos de viento y tahonas (15).

\section{La VILA NOVA}

Después de la conquista castellana, Alicante mantuvo las funciones y el carácter urbano del primitivo recinto musulmán, es decir, el de la

(9) J. M. DEL ESTAL, «Castrum d'Alacant», ITEM, 5, p. 58; Provisión Real de Pedro IV el Ceremonioso en favor de la villa de Alicante, AMA, Arm. 1, tib. 2, fol. 3v. (Barcelona, 1366).

(10) J. TORRES FONTES, CODOM III, dto. LV, pp. 74-75. Cordoba, 30 de junio de 1260.

(11) J. M. DEL ESTAL, op. cit., p. 90.

(12) CODOM III, pp. 17, 39, 59, 60, 75, 76 y 108.

(13) CODOM III, dto. IX, p. 78.

(14) CODOM III, dto. LIV, p. 73, Córdoba 29 de junio de 1260; cf. MARTínEZ MORELLA, op. cit., P. 28; AMA Arm. 5, L, 48, p. 25v.

(15) V. MARTÍNEZ MORELLA, op. cit., p. 21; AMA, Arm. 5, L. 48, fol. 17 (Doc. Junio 1258). 
pequeña urbe portuaria a la que el castillo concedía gran interés estratégico, aunque la llegada de los repobladores tendría, no obstante, una influencia y repercusiones decisivas para la expansión de la ciudad por la creación de nuevos espacios construidos al servicio de éstos (16).

Como consecuencia de la separación espacial de las comunidades los musulmanes quedarían relegados a ocupar los peores sectores de la ciudad, los menos accesibles de la vila vella, en lo más alto de la ladera del Benacantil, en la Moreria (17) que aún se cita en la documentación de finales del siglo XIV, en donde las viviendas, de menor tamaño y valor, pagarían impuestos notablemente inferiores a los que soportaban los otros habitantes de la ciudad, aunque en las anotaciones fiscales sobre los tipos de actividad de los siglos XIII y XIV se observa una similitud de oficios entre una y otra zona.

Desde la conquista se amplió el recinto islámico de la vila vella con una vila nova que iría extendiéndose, fuera del perímetro de la antigua muralla de la ciudad, por el llano costero inmediato, localizado a poniente del Benacantil, ocupando, de esta manera, una franja envolvente de dirección NW.-SE., cuyos límites resultaban de nuevo afianzados por la topografía cuando se construyera el muro defensivo sobre el inmediato cauce de la rambla de Canicia que le serviría de foso y límite urbano occidental hasta el siglo XVI.

La primera alusión a esta ampliación de la ciudad se encuentra en el Llibre de/s feits del rey D. Jaime I (18), donde se menciona la iglesia novella de fora (de fuera del recinto musulmán) en la que el rey reunió a sus nobles para tratar de la conquista militar de Murcia. En este sentido, pero más ricas en información son las referencias de la Crónica de R. Muntaner a la toma militar de Alicante y su castillo por Jaime Il en las operaciones bélicas de la anexión valenciana de los territorios meridionales del Reino que insisten, asimismo, en la descripción de la dualidad urbana, ya existente en este momento, entre la vila vella y la nueva ciudad (19). Insistimos finalmente en este hecho haciendo mención del documento notarial de Thomas Pina, quien a voluntad testamentaria de Bernardo Gomis, registra las cláusulas de la donación por

(16) V. GOZÁlVEZ, La ciudad de Elche, p. 43.

(17) A.R.V. Real Patrimonio, Maestre Racional 4543, año 1376.

(18) Apud. F. SOLDEVILA, Les Quatre grans cróniques, Crónica C 415, p. 151:... en l'esglesia d'Alacant, en la novella de fora, no en la major....

(19) R. MUNTANER: Crónica, c. 188, Ed. 62, 1979. 
éste de un hospital para la ciudad, signado asimismo en "la vila nova d'Alacant" (20).

En este nuevo espacio construido fue creciendo el caserío, abriéndose nuevas calles como la de Labradores y la Mayor, al final de la cual se construyeron dos torres que flanqueaban la puerta de la muralla de salida al camino de Elche (21), que ha dejado expresión toponímica en la plaza que se abre inmediatamente delante de la intersección de la calle Mayor con la Rambla. Algunas de estas calles suponían la incorporación urbana de algunos caminos de extramuros: carrera de Elche, camino de la huerta de Sueca, situada en las cercanias, al W. de la ciudad o aseguraban la articulación de la nueva trama urbana.

Aquí construyó sus viviendas la nueva clase dirigente de la ciudad, como lo harían los Morales de Finestrat, o los caballeros aragoneses del rey $D$. Jaime II que después de conquistar de nuevo la ciudad en julio de 1296 se establecieron en el sector comprendido entre las calles de San Agustín y la Rambla, espacio urbano en el que se iba edificando desde la época de Alfonso el Sabio (22).

Como resultado de ello la vila nova se configuró al modo de las ciudades medievales levantinas de nueva fundación (23), con una trama ortogonal en la que destacaban los ejes principales de las dos calles antes citadas: la de Labradores, trazada con dirección meridiana hacia el mar y en la que se ubicó la iglesia de San Nicolás, y la Mayor, perpendicular a aquélla, trazada desde el Portal de Elche, principal vía de acceso - desde la vila nova - al recinto de la vila vella.

Estas y sus complementarias presentan un trazado geométrico diferente del que puede observarse en la ciudad musulmana, más adaptado a la topografía del cerro, produciendo, a la vez, un conjunto de manzanas de mayor tamaño y simetría; todo ello constituyen diferencias formales que permiten individualizar cartográficamente ambos espacios urbanos (24).

(20) V. BENDICHO: Crónica de la Muy llustre, Noble y Leal Ciudad de Alicante. Alicante, 1640. Ed. resumida y anotada por F. FIGUERAS PACHECO, Alicante I.E.A., 1960, p. 61.

(21) R. VIRAVENS Y PASTOR: Crónica de Alicante, p. 26.

(22) F. FIGUERAS PACHECO: Compendio historico de Alicante, p. 134.

(23) A. GARCIA Y BELLIDO, L. TORRES BALBAS et al.: Resumen histórico del urbanismo en España, p. 121 y ss.

(24) M. BEVIA: op. cit., p. 72 y ss. 


\section{LAS OBRAS DE DEFENSA}

Las escasas referencias que nos han llegado en relación a las obras de defensa de la ciudad, en parte, quedaron ya consignadas con lo dicho al referirnos a la construcción de la muralla que ceñía la villa nueva alfonsina (25). Por otra parte, sabemos que Jaime II las mejoró, dado su interés en conservar Alicante (26), que en tiempos de Alfonso IV (27) se rehicieron fortificaciones y que tras la guerra castellano aragonesa, en tiempos de Pedro IV (28), volvieron a mejorarse. De estos últimos refuerzos existe mención expresa, de 1376, a la torre de l'Esperó (29), situada a espaldas de la iglesia de Santa María junto al Portal Nou, del que encontramos mención diez años más tarde (30). Este abría una salida al sector oriental extramuros de la ciudad, limitado por la Rambla de Bonivern, donde existía un pozo de agua dulce, el Pou del Drac, y el manantial de la Goteta, además de los eremitorios de la Virgen del Lluc y el de Santa Ana, lugar en el que se desarrollaría, siglos más tarde, el llamado arrabal Roig.

La constante mención de los "obrers de murs y valls" indica la preocupación por el buen estado de las murallas, cuyo trazado, aprovechando el del lienzo árabe que descendía del castillo, al E. de la ciudad, se desarrollaba paralelo al mar, desde la Torre de L'Esperó en dirección W., ciñendo la vila vella por el costado meridional de la iglesia de Santa María y la vila nova, hasta la rambla de Canicia, cuya margen izquierda remontaba, con un recorrido, en este último tramo, paralelo al que llevaría la posterior muralla renacentista. Este dato se refuerza por la existencia de una calle, paralela a la de Labradores, llamada "de Entre Muro y Muro", trazada entre los cuerpos de ambas murallas, cuya finalidad era enlazar con el muro antiguo que en las estribaciones del Benacantil descendia desde el castillo hacia la puerta Ferrisa. Queda por dilucidar de dicho recorrido el trazado preciso que seguiría el resto de muralla que abarcara el recinto de la vila nova.

A lo largo de dicho circuito se abrían tres puertas conocidas: el men-

(25) Véase nota 13.

(26) F. FIGUERAS PACHECO: op. cit, nota 22.

(27) N.C. JOVER: Reseña histórica de la ciudad de Alicante, p. 34.

(28) F. FIGUERAS PACHECO: Geografia de la provincia de Alicante, p. 477.

(29) A.R.V., Real Patrimonio, Maestre Racional, 1543 (año 1376).

(30) A. ARQUES JOVER: Nobiliario alicantino. Transcripción, adiciones, notas y comentarios de Luis Mas y Gil y J. M. Esquerdo Ribera, p. 122, citado por M. BEVIA: op. cit, p. 60. 
cionado Portal Nou, el portal de N." S.a de Monserrat, de salida al muelle desde la plaza de la Fruita (actual Santa Faz) y el Portal de Elche. Acaso cabe mencionar otro más, el de la Huerta, al N., en las cercanías de la conjunción del lienzo defensivo occidental con el $\mathrm{Be}$ nacantil.

\section{EDIFICIOS URBANOS MAS DESTACADOS}

El ámbito urbano que describimos adensaría paulatinamente su trama con edificios propios del servicio interno de la ciudad y con aquellos necesarios al desenvolvimiento de la actividad comercial y portuaria de la misma, conservándose la memoria de los más significativos.

Es sabido que en el reinado del aragonés Alfonso IV, a la vez que se construyeron nuevos edificios y se rehicieron las fortificaciones (31), se levantó un hospital, fundado por Bernardo Gomis, según testamento otorgado el 25 de abril de 1333, llamado de San Juan Bautista, hasta trescientos años más tarde en que recibió la denominación de San Juan de Dios. Estaba situado en la vila nova, en terrenos donde, tras su demolición, a mediados del pasado siglo, se abrió la calle de Montengón y cuyos pozos de enterramiento se descubrieron al realizarse las obras del colector de la ciudad en 1910 (32). Su ubicación precisa, conocida por una frase del texto notarial del testamento citado, limitaba al W. con la actual calle de San Nicolás y por el E. con su paralela, la de Fontanella (actual San Agustín) (33).

Como antecedente de dicho hospital se indica la existencia de otro más antiguo, que acaso fuese un elemental albergue, fundado por los Hermanos de San Antonio Vienés en la Villavieja, junto a la iglesia de Santa María (34). Además, y por lo que respecta a los establecimientos hospitalarios, podemos añadir que existe constancia documental, de mayo de 1264, de otro hospital, cercano al cementerio de moros "situado en la carrera de Murcia, junto al barranco" (Rambla de Méndez Núñez) (35).

A consecuencia de la Guerra de los dos Pedros la villa quedó arra-

(31) N. C. JOVER: op. cit., p. 34.

(32) F. FIGUERAS PACHECO: Op. cit., en nota 28, p. 476.

(33) R. MARTINEZ SANPEDRO: Historia de los Hospitales de Alicante, p. 34.

(34) R. MARTÍNEZ SANPEDRO: Op. cit., p. 27.

(35) J. TORRES FONTES, CODOM II, pp. 19-20, Doc. 1264. V-19. 
sada, inútiles sus fortificaciones, las casas en ruinas, los moradores mutilados, los campos talados, el puerto perdió su tráfico y la ciudad quedó yerma y despoblada, como queda descrito por el canciller Pero López de Ayala (36). Por ello es deducible una cierta actividad reconstructora ulterior. En tiempos de Pedro IV se rehizo la ciudad, se repararon las murallas (37) y se activó el comercio, autorizándose la construcción de una Lonja de Contratación, predecesora de la que se levantaría en el sigio XV en las cercancías del edificio del actual Ayuntamiento, entre las plazas de la Fruita y la de las Monjas, realzando el carácter comercial de aquella plaza que acaso sirviera de antiguo zuq a la vila nova, dada su situación ante el portal de N. ${ }^{a}$ S. ${ }^{a}$ de Montserrat, en el límite de la nueva ciudad y conectada, a través de la puerta Ferrisa, con la Villavieja (38).

Dos eran las construcciones religiosas más notables de la ciudad: la gótica iglesia Mayor (39), dedicada a Santa María, edificada, sobre el espacio ocupado por la antigua mezquita principal, en la Villavieja, con diseño de los arquitectos de la escuela de Morella, que fue fundada por el rey $\mathrm{D}$. Jaime para dotar de edificios públicos e iglesias a todo el reino y que aún conserva trazas de su primitiva estructura, pese a las varias reformas sufridas, especialmente las obligadas tras el incendio de 1484 (40). La segunda de éstas fue la iglesia gótica de San Nicolás (41), erigida en el solar del antiguo cementerio de moros, ubicado en el lugar hoy ocupado por la Capilla de la Comunión de la iglesia Concatedral. Dicha iglesia sería también de nueva obra, frente a la opinión de quienes la consideran antigua mezquita dedicada al culto cristiano o, incluso, anterior. El acierto de considerarla obra nueva se ve corroborado por los textos del Rey Conquistador, en los cuales se cita como «la novella», «la de fora», frente la que estaba en el interior, «la anti-

(36) LOPEZ de AYALA, Crónicas de los Reyes de Castilla, Crónica de D. Pedro I, BAE LXVI, p. 498.

(37) F. FIGUERAS PACHECO: Op. cit., en nota 22, p. 144.

(38) R. VIRAVENS Y PASTOR: Op. cit., p. 37.

(39) R. MARTÍNEZ SANPEDRO, op. cit., p. 27; V. MARTÍNEZ MORELLA, «EI hospital de peregrinos y enfermos de Alicante, a cargo de los canónigos antonianos». VIII Congr. de Hist. de la Corona de Aragón, 1969, T. II, vol. 1, p. 92; R. I. BURNS, Jaume li els valencians del s. XIII, p. 20.

(40) S. VARELA BOTELLA y J. CALDUCH CERVERA: Guia de arquitectura de Alacant, T. II, p. 15.

(41) F. SOLDEVILA: vid. nota 18. 


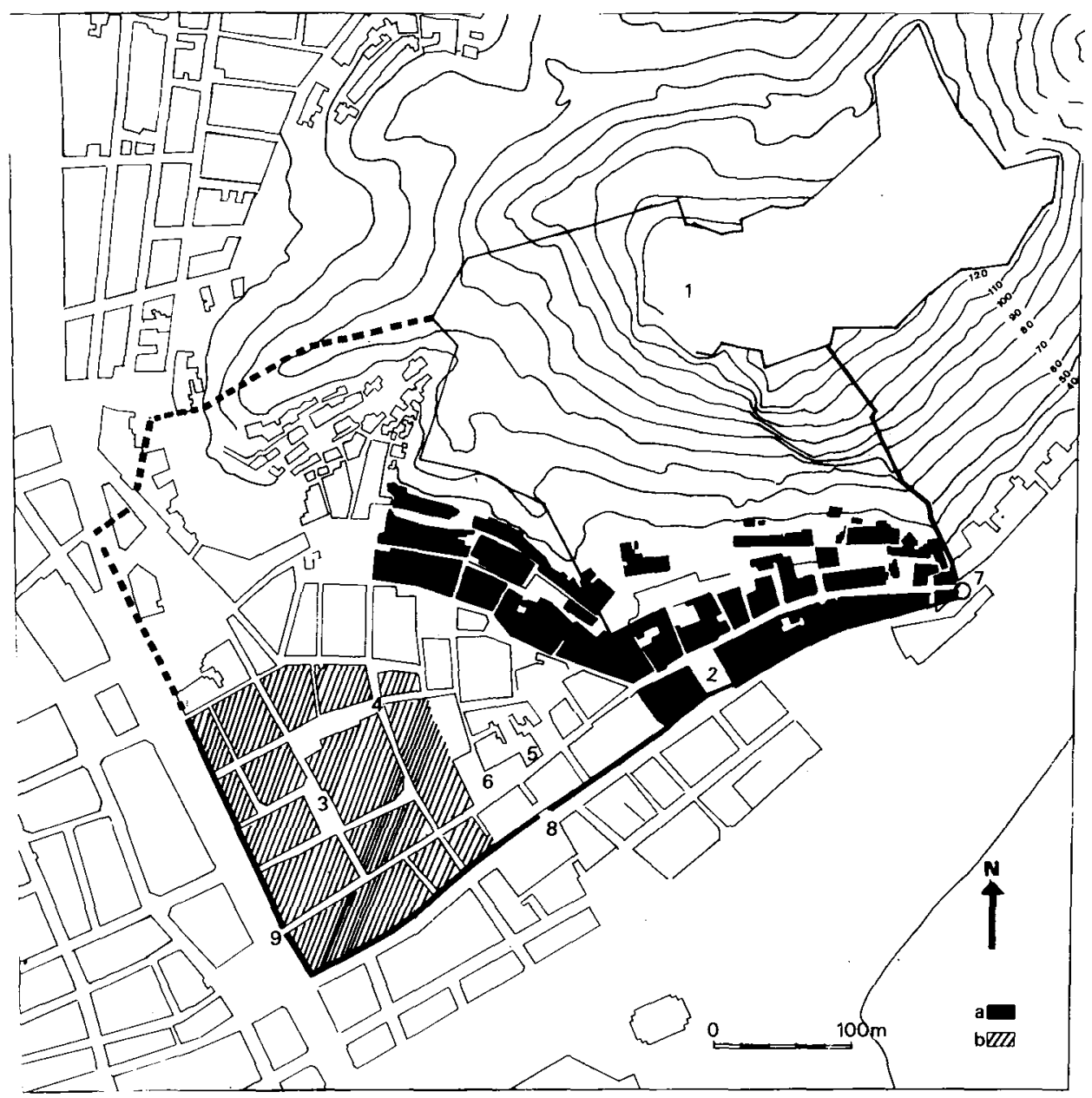

La ciudad de Alicante tras la conquista castellano-aragonesa. En negro: recinto musulmán con la Villa vieja; en trazos, la vila nova.

1: Castillo

2: Iglesia de Santa Maria

3: Iglesia de San Nicolás

4: Hospital

5: Lonja

6: Plaza de la Fruta

7: Espolón y Portal Nou

8: Puerta del Mar

9: Puerta de Elche 
gua», «la Mayor" (Santa María), que aluden a su construcción, inmediatamente posterior a la conquista. En ella se celebraron las sesiones del Concejo Municipal (42) hasta que en 1370 se construyó la primitiva Lonja, lugar a donde se trasladaron. Ampliada en diversas etapas, a principios del siglo XIV se le añadió una esbelta torre campanil que subsistió hasta 1634, en que fue derribada al construirse la actual iglesia (43).

\section{LA POBLACION}

Las dificultades para conocer el número de habitantes de la época preestadística son enormes, dado que hay que trabajar sobre conjeturas peor o mejor fundadas. Las relaciones de hogares hechas con miras fiscales para el reparto de cargas tributarias suelen adolecer de un carácter restrictivo y normalmente excluye a los pobres, imposibilitados de pagar, y a las clases exentas, la nobleza y el clero, con lo que no se puede fijar el número exacto de familias ni el de los habitantes. Con todo, son las únicas fuentes de las que podemos deducir un perfil de la evolución poblacional (44).

Para el Alicante medieval disponemos de las series informativas extraídas del pago del impuesto del morabatí que abarca desde finales del siglo XIV (1373) hasta 1499, en las que podemos observar el lento crecimiento del número de vecinos y del total de habitantes calculados (45). El número de habitantes puede guardar proporción con el establecido para la época musulmana, según registramos en el siguiente cuadro:

\section{CUADRO I}

\begin{tabular}{ccc}
\hline Años & Vecinos & Habitantes \\
\hline 1373 & 422 & 2100 \\
1439 & 468 & 2340 \\
1499 & 602 & 3010 \\
\hline
\end{tabular}

(42) F. SALA SEVA: Acontecimientos notables en la lglesia de San Nicolás de Alicante, 1245 a 1980, p. 25.

(43) F. SALA SEVA: Op. cit., p. 36.

(44) P. PEREZ PUCHAL, "La población del País Valenciano hasta la época estadística", Cuad. de Geogr., 10, Valencia, 1972; p. 1.

(45) P. M. ORTS y $\mathrm{BOSCH}, \mathrm{Op}$. cit., p. 15 y ss. 
Sin duda este crecimiento debe colacionarse con las crisis de mortalidad propias de estos siglos medievales. En 1299 (46) fue el hambre y tenemos noticias de peste en los siguientes años: 1340 y 1375, 1394 y 1411. Aunque desde 1484 se empezó a poner en uso en el puerto el método de la cuarentena para evitar los contagios, ello no fue posible. De nuevo, la peste, en 1489, acometió a la ciudad. Martínez Sanpedro, con relación al estado sanitario de la población, cita la existencia de otras enfermedades y contagios sufridos también durante los últimos años del siglo XV (47).

(46) R. MARTINEZ SANPEDRO, Apuntes históricos sobre las epidemias en Alicante, pp. 11 y ss.

(47) R. MARTÍNEZ SANPEDRO: Op. cit., en nota 46, p. 14. 